\title{
Algunos errores numéricos en la respuesta del estado estable de sistemas mecánicos vibratorios
}

\section{Some numerical errors in the steady state response of vibratory mechanical systems}

VÁZQUEZ-GONZÁLEZ, Benjamín†*, JIMÉNEZ-RABIELA, Homero, RAMÍREZ-CRUZ, José Luis y BRAVO-ACOSTA, Adrian Gustavo

Universidad Autónoma Metropolitana, Unidad Azcapotzalco, División de Ciencias Básicas e Ingeniería, Departamento de Energía, Av. San Pablo 180 Colonia Reynosa Tamaulipas, Azcapotzalco C. P. 02200 Ciudad de México, México

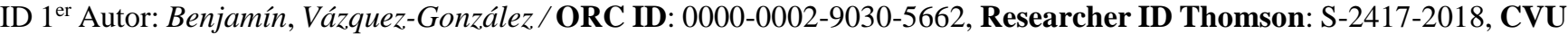
CONACYT ID: 25749

ID $1^{\text {er }}$ Coautor: Homero, Jiménez-Rabiela / Researcher ID Thomson: S-2299-2018, CVU CONACYT ID: 123386

ID $2^{\text {do }}$ Coautor: José Luis, Ramírez-Cruz / ORC ID: 0000-0003-0762-2630, Researcher ID Thomson: G-3405-2019, CVU CONACYT ID: 921268

ID $3^{\text {er }}$ Coautor: Adrian Gustavo, Bravo-Acosta / ORC ID: 0000-0001-57975317, Researcher ID Thomson: 2272-2018, CVU CONACYT ID: 334391

DOI: $10.35429 / \mathrm{JME} .2019 .12 .3 .1 .9$

Recibido 08 Agosto, 2019; Aceptado 30 Noviembre, 2019

\section{Resumen}

En este trabajo se presentan resultados sobre la simulación numérica de la respuesta en el estado estable de la vibración de sistemas mecánicos lineales y errores que se pueden presentar con los algoritmos con los que se realiza la simulación. Para algunos parámetros numéricos de un sistema mecánico vibratorio amortiguado y no amortiguado, el resultado de la simulación numérica no es consistente con la definición de estado estable, ésta falta de congruencia con lo observado no se detecta fácilmente cuando el desempeño del sistema se realiza para altas frecuencias, debido a que para altas frecuencias de excitación, las amplitudes de respuesta del sistema son muy pequeñas. Se realiza un análisis de la respuesta en el tiempo, empleando métodos numéricos tradicionales contenidos en sistemas de cómputo con lenguajes de programación propios, detectando el mismo tipo de error, dependiendo del método numérico empleado. El análisis de la vibración forzada de sistemas mecánicos vibratorios no lineales, tiene un desempeño semejante al sistema lineal para un rango determinado de frecuencias, por lo que determinar de manera correcta las respuestas en el estado estable del sistema lineal, es fundamental para estudios posteriores, parte del análisis correcto depende del algoritmo numérico que se elija utilizar.

Estado estable, Métodos numéricos, Altas frecuencias

\begin{abstract}
In this work numerical simulation results are shown for the steady state response of linear mechanical oscillating systems and the numerical errors that can be present when some numerical algorithms are used to perform the simulations. For some numerical parameters, the mechanical oscillating system with or without damping response, does not converge to the expected steady state response; this discrepancy is not easily detected when the performance of the system is on the range of high excitation frequencies, due that for of high excitation frequencies the amplitude in the steady state response reaches very small values. We perform the time response of the system using conventionally numerical methods included in the common programming platforms, and the result is that using the same algorithm in different platforms the error is the same; selecting other numeric algorithm the result in satisfactory. Non linear forced mechanical vibration systems; behave like linear systems for some frequency range, then is very useful to obtain the right or correct responses in the steady state response for the linear system, this is fundamental for forward studies, the right analysis is based on the selected numeric algorithm.
\end{abstract}

Steady state, Numerical Methods, High frequencies

Citación: VÁZQUEZ-GONZÁLEZ, Benjamín, JIMÉNEZ-RABIELA, Homero, RAMÍREZ-CRUZ, José Luis y BRAVOACOSTA, Adrian Gustavo. Algunos errores numéricos en la respuesta del estado estable de sistemas mecánicos vibratorios. Revista de Ingeniería Mecánica. 2019. 3-12: 1-9

\footnotetext{
* Correspondencia al Autor (Correo electrónico: bvg@correo.azc.ua.mx)

$\dagger$ Investigador contribuyendo como primer Autor.
} 


\section{Introducción}

La vibración mecánica es un fenómeno que se presenta en la realidad cotidiana, su percepción es evidente para los individuos cuando se manifiesta dentro del rango de la sensibilidad de quien la registra. La vibración mecánica se presenta como un fenómeno que puede ser destructivo, pero si se le estudia y comprende, entonces se puede convertir en una herramienta constructiva. La vibración mecánica presenta fundamentalmente dos características básicas; su amplitud y su frecuencia. Son muchas las situaciones en las que los modelos que describen el comportamiento vibratorio de un sistema mecánico, se ajustan de manera precisa a la respuesta observada en la realidad.

A grandes rasgos, la vibración mecánica se puede clasificar cómo vibración lineal o bien, no lineal. Bajo determinadas condiciones de desempeño y también, dependiendo de la naturaleza de los parámetros que definen a las ecuaciones, se puede tener una convergencia de la vibración no lineal a la lineal.

Una herramienta fundamental en el estudio de las vibraciones mecánicas es la simulación realizada por medio de métodos numéricos. Recientemente los métodos numéricos han evolucionado mucho, gracias a los desarrollos informáticos como capacidad de almacenamiento en memoria o velocidad de procesamiento, sin embargo, los fundamentos de análisis de la vibración, siguen estando vigentes, sin embargo, la realización de experimentos también proporciona buenas referencias, ver por ejemplo, León, R. A. et. al (2019).

En las últimas décadas también los métodos denominados de perturbaciones, se han empleado en el estudio de las vibraciones no lineales, no obstante los métodos numéricos tradicionales siguen siendo un referente importante para evaluar las respuestas asociadas a los comportamientos vibratorios. Los métodos de perturbaciones permiten obtener expresiones analíticas de sistema no lineales. En este trabajo se hace resaltar la importancia de contar con un referente concreto, a fin de validar los resultados obtenidos por medio de simulaciones numéricas. En este trabajo se presentan casos particulares en los que se utilizan métodos numéricos, que discrepan con los resultados analíticos sobre la vibración forzada amortiguada.
Así mismo, se logra establecer la razón por la que se presenta dicha discrepancia.

\section{Fundamentos de la vibración forzada mecánica lineal}

La vibración mecánica lineal forzada corresponde con el movimiento repetitivo que desarrolla un cuerpo rígido, cuyo centro de masa se ve sujeto a una acción restitutiva y a una acción que induce su movimiento o excitación armónica, en la que la amplitud del movimiento queda establecida por la magnitud de la excitación armónica. La frecuencia del movimiento del cuerpo es constante y corresponde con la frecuencia de excitación. La frecuencia de movimiento del cuerpo podrá presentar un desfasamiento en relación con la acción que produce su movimiento, si existe amortiguamiento. En la práctica el amortiguamiento es una acción inherente, sin embargo, hay condiciones en las que puede adquirir valores muy pequeños del mismo.

Esta descripción muy simplificada de la vibración forzada lineal es cierta para cada valor de la frecuencia de excitación. También es importante establecer la forma en la que el movimiento, descrito por la amplitud que desarrolla el cuerpo, cambia en términos de las distintas frecuencias a las que se ve sujeto. Para ilustrar el fenómeno de la vibración mecánica lineal forzada, considérese la siguiente figura,

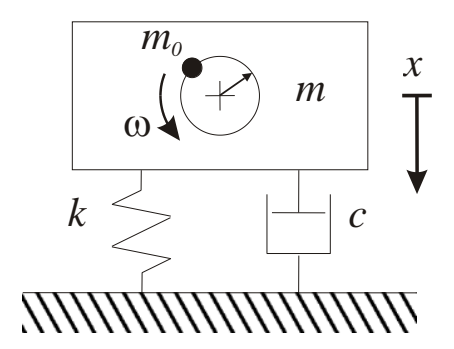

Figura 1 Sistema masa-resorte-amortiguador excitado armónicamente

En la Figura 1, se tiene una cuerpo rígido de masa $m$ sujeto a la acción de una fuerza de tipo armónico inducida por la rotación de la masa $m_{0}$, a una velocidad angular $\omega$, el sistema mecánico se encuentra sobre materiales que tienen un comportamiento elástico y a su vez de tipo viscoso, lo cual se representa por medio de un resorte $k$ y un amortiguador $c$ respectivamente. 
La ecuación de movimiento se determina por medio de la segunda ley de Newton, el resultado es,

$$
m \ddot{x}+c \dot{x}+k x=\left(m_{0} r \omega^{2}\right) \operatorname{sen} \omega t
$$

donde $r$ representa la distancia o desbalance de la masa $m_{0}$ (también se le denomina excentricidad) que induce fuerzas centrípetas.

Cuando se normaliza la ecuación (1) resulta la siguiente ecuación,

$\ddot{x}+2 \zeta \omega_{n} \dot{x}+\omega_{n}^{2} x=\left(\frac{m_{0} r \omega^{2}}{m}\right) \operatorname{sen} \omega t$

en donde,

$\frac{c}{m}=2 \zeta \omega_{n}, \omega_{n}=\sqrt{\frac{k}{m}}$

$\mathrm{Al}$ parámetro $\zeta$ se le denomina cociente de amortiguamiento.

La ecuación diferencial (2) está expresada en términos de la frecuencia de excitación $\omega$ y de la frecuencia natural del sistema $\omega_{n}$. Esta ecuación diferencial tiene por solución la siguiente expresión,

$x(t)=\frac{\left(\frac{m_{0} r \omega^{2}}{m}\right)}{\sqrt{\left(\omega_{n}^{2}-\omega^{2}\right)^{2}+\left(2 \zeta \omega \omega_{n}\right)^{2}}} \operatorname{sen}(\omega t-\phi)$

donde,

$\phi=\arctan \left(\frac{2 \zeta \omega \omega_{n}}{\omega_{n}^{2}-\omega^{2}}\right)$

A este término se le denomina ángulo de desfasamiento y establece una medida del movimiento del sistema, en relación con la acción que actúa sobre la masa $m$.

Para evaluar el comportamiento del sistema mecánico en términos de la frecuencia, ahora se expresa la ecuación (5) en función del cociente de las frecuencias presentes, el resultado es el siguiente,

$$
x(t)=\frac{\left(\frac{m_{0} r}{m}\right)\left(\frac{\omega^{2}}{\omega_{n}^{2}}\right)}{\sqrt{\left(1-\left(\frac{\omega}{\omega_{n}}\right)^{2}\right)^{2}+\left(2 \zeta \frac{\omega}{\omega_{n}}\right)^{2}}} \operatorname{sen}(\omega t-\phi)
$$

La amplitud del movimiento corresponde con la expresión,

$$
X=\frac{\left(\frac{m_{0} r}{m}\right)\left(\frac{\omega^{2}}{\omega_{n}^{2}}\right)}{\sqrt{\left(1-\left(\frac{\omega}{\omega_{n}}\right)^{2}\right)^{2}+\left(2 \zeta \frac{\omega}{\omega_{n}}\right)^{2}}}
$$

A la ecuación (7) se le conoce cómo respuesta en frecuencia, ver por ejemplo, Meirovitch (2001), en el que se presenta un descripción un poco más detallada de solución, o bien el libro de Ginsberg (2001), que presenta una solución más elaborada al utilizar la variable compleja para determinar la respuesta en frecuencia.

La ecuación (7) contiene toda la información relativa al comportamiento de la amplitud del sistema, en términos de la frecuencia de excitación, $\omega$ y en relación con la frecuencia natural $\omega_{n}$.

\section{El estado estable}

En esta sección se establece la forma en la que se relacionan la respuesta en el tiempo, en el estado estable y la respuesta en el espacio de la frecuencia.

Meirovitch (2001), también acude a la solución por medio de la variable compleja para obtener una respuesta en frecuencia y definir el estado estable, éste libro como el de Ginsberg (2001), también define otros términos, tal como la función de impedancia, que corresponde con la velocidad compleja a partir de la amplitud del estado estable.

La definición de estado estable corresponde con una región del comportamiento en el tiempo, a partir de la cual, la amplitud adquiere un comportamiento regular, es decir, el rango de la amplitud de vibración se desempeña con una señal armónica y de amplitud constante.

En la Figura 2, se muestra una gráfica de la ecuación (7) y corresponde con la respuesta de la amplitud en términos del cociente de frecuencias $\frac{\omega}{\omega_{n}}$. En esta gráfica se han incluido algunos valores del cociente de amortiguamiento $\zeta$. Hay que resaltar que la amplitud registrada en esta gráfica de la frecuencia, corresponde con la respuesta en el estado estable o también denominado estado permanente del tiempo. 


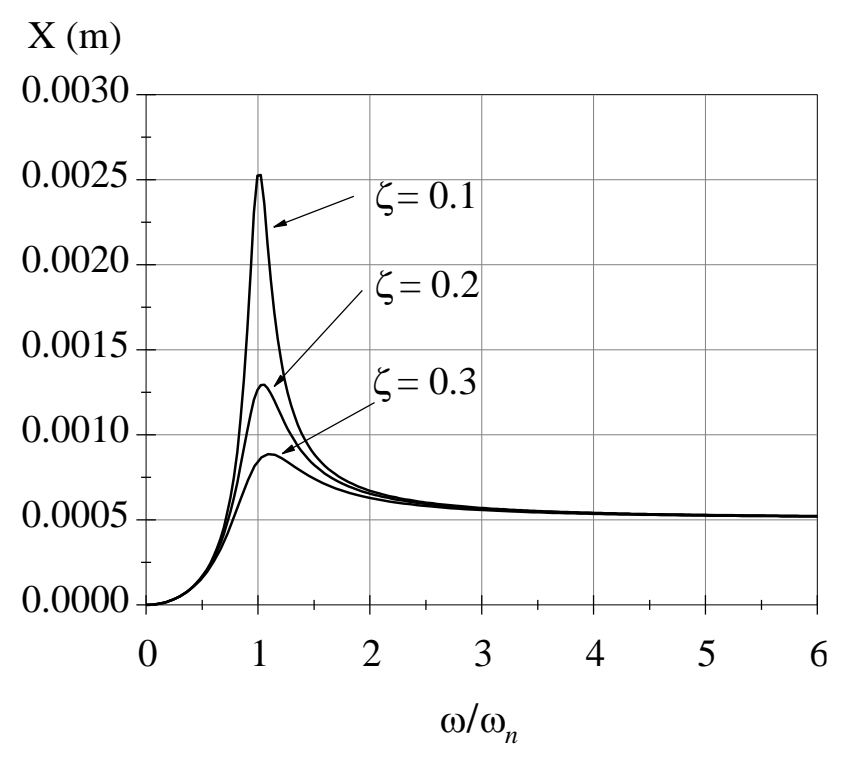

Figura 2 Respuesta en frecuencia de la amplitud de vibración de un sistema mecánico forzado

Otros valores numéricos correspondientes a la gráfica de la Figura 2 se muestran en la Tabla1, siguiente,

\begin{tabular}{|l|l|}
\hline $\mathbf{m}_{0} \mathrm{r}=0.2303[\mathrm{kgm}]$ & $\mathbf{m}=453.4[\mathrm{~kg}]$ \\
\hline$\zeta_{1}=0.1$ & $\zeta_{3}=0.3$ \\
\hline$\zeta_{2}=0.2$ & \\
\hline
\end{tabular}

Tabla 1 Valores numéricos de los parámetros del sistema masa-resorte amortiguado para la Figura 2

De la gráfica de la Figura 2 se puede determinar, por ejemplo, que para un sistema mecánico con un cociente de amortiguamiento con un valor de $\zeta=0.2$ y un cociente de frecuencias $\frac{\omega}{\omega_{n}}=1.3$, la amplitud $X$ de la vibración tendrá un valor de $X=0.001 \mathrm{~m}$, en el estado estable.

El análisis de amplitud en términos de la frecuencia da pautas muy importantes para comprender completamente el desempeño del sistema. En un primer acercamiento a ésta gráfica, se le puede estudiar definiendo tres sectores acotados por las abscisas, es decir, por el cociente de frecuencias $\frac{\omega}{\omega_{n}}$.

El primer sector se establece para valores de las abscisas en el rango $0 \leq \frac{\omega}{\omega_{n}}<0.5$, el segundo sector, 0. $5 \leq \frac{\omega}{\omega_{n}}<2.5$ y finalmente el tercer sector $2.5 \leq \frac{\omega}{\omega_{n}}$.
Esta definición de sectores se realiza al observar que en los sectores uno y tres, la variación de la amplitud es relativamente pequeña, para cada valor del cociente de amortiguamiento, pero independientemente del mismo, incluyendo el caso no amortiguado, es $\operatorname{decir} \zeta=0$, por ejemplo, para una relación de frecuencias $\frac{\omega}{\omega_{n}}=0.5$ la amplitud tendrá un valor de $X=0.00016727 \mathrm{~m}$, sin poder observar con claridad, qué cociente de amortiguamiento le corresponde.

En la región dos, se tienen grandes variaciones de la amplitud para cambios pequeños en el cociente de amortiguamiento.

La región de interés en este trabajo corresponde al sector tres, en el que la relación de frecuencias es $\frac{\omega}{\omega_{n}} \geq 2.5$. A partir de este valor y conforme la relación de frecuencias aumenta, los valores de amplitud cada vez se asemejan más entre sí, hasta adquirir prácticamente el mismo valor numérico, sin importar el valor numérico del cociente de amortiguamiento $\zeta$.

Lo anterior corresponde a un caso numérico particular, sin embargo, el comportamiento general está descrito por la misma expresión y las variaciones de amplitud definidas, establecen las tres regiones mencionadas.

La ecuación (7) se vuelve una referencia concreta, que permite conocer con muy buena aproximación, la amplitud de la solución de la ecuación diferencial, correspondiente para un sistema mecánico vibratorio, cuando se conocen los valores de todos los parámetros que definen al movimiento. En esta sección se han definido, a grades rasgos tres zonas en las que se puede dividir la respuesta en frecuencia, siendo de interés la región de altas frecuencias.

\section{Determinación de la amplitud por simulación}

Actualmente son muchos los estudios que se realizan en relación con la resonancia, esto coincide con el sector dos que se definió en la sección anterior, pero son menos los estudios realizados para altas frecuencias de sistemas mecánicos lineales vibratorios. Chunling (2019), menciona algunas técnicas para el análisis de algunos problemas en vibraciones a altas frecuencias. 
Emerson (2017), presenta un trabajo en el que se denota la importancia del estudio de las vibraciones mecánicas a altas frecuencias. En esta sección se presenta una serie de experimentos numéricos, que representan la vibración lineal forzada de un sistema mecánico, donde se obtiene la respuesta en el tiempo y ésta se compara con la respuesta obtenida por medio del análisis en la frecuencia.

La respuesta en el estado estable correspondiente a los valores de la Tabla 2 , se muestra a continuación en la gráfica de la Figura 3. Esta gráfica se ha obtenido con el simulador por computadora SIMNON para Windows ${ }^{\circledR}$, con el método Runge-Kutta-Fehlberg 4/5 (RKF4/5). Este programa fue desarrollado por Åström (1982).

\begin{tabular}{|l|l|}
\hline $\mathrm{m}_{0} \mathrm{r}=0.2303[\mathrm{kgm}]$ & $\mathrm{m}=453.4[\mathrm{~kg}]$ \\
\hline$\zeta=0.01$ & $\omega_{\mathrm{n}}=44[\mathrm{rad} / \mathrm{s}]$ \\
\hline$\omega=3 \omega_{\mathrm{n}}[\mathrm{rad} / \mathrm{s}]$ & \\
\hline
\end{tabular}

Tabla 2 Valores numéricos de los parámetros del sistema masa-resorte amortiguador para la Figura 3

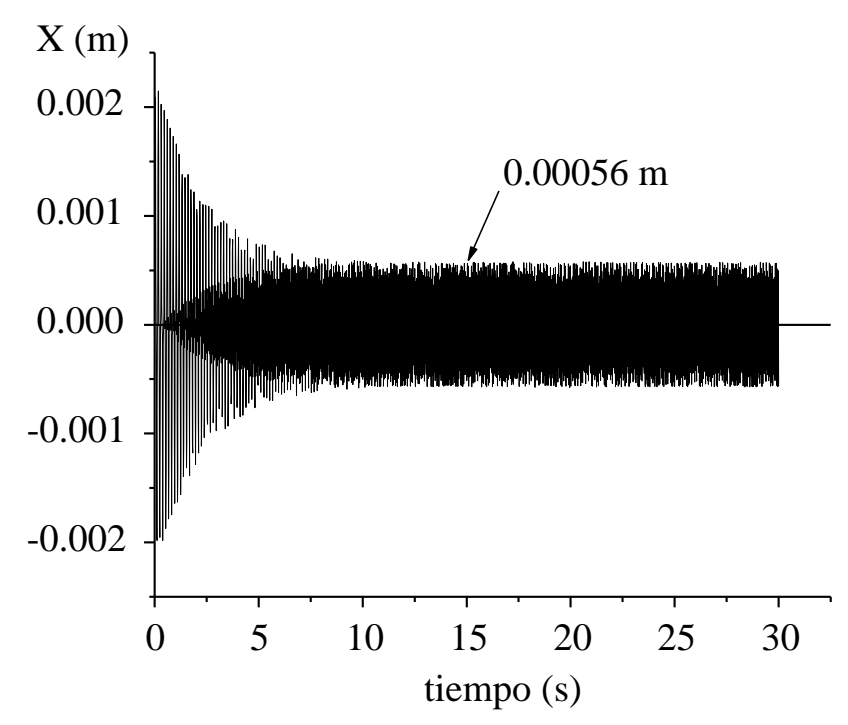

Figura 3 Respuesta en el estado estable para los datos de la Tabla 2

En la gráfica de la Figura 3, se aprecia el comportamiento esperado de la respuesta en estado permanente con amortiguamiento. Se puede observar de la gráfica, que la amplitud del estado estable tiene un valor de $X=0.00056 \mathrm{~m}$. Ahora considérense los valores numéricos de la Tabla 3 siguiente,

\begin{tabular}{|l|l|}
\hline$\omega=3 \omega_{\mathrm{n}}[\mathrm{rad} / \mathrm{s}]$ & $\mathrm{m}_{0} \mathrm{r}=0.2303[\mathrm{kgm}]$ \\
\hline$\omega_{\mathrm{n}}=44[\mathrm{rad} / \mathrm{s}]$ & $\mathrm{m}=453.4[\mathrm{~kg}]$ \\
\hline$\zeta=0.008$ & \\
\hline
\end{tabular}

Tabla 3 Valores numéricos de los parámetros del sistema masa-resorte amortiguador para la Figura 4
A continuación, en la Figura 4 (RKF4/5) se presenta la simulación de la respuesta en el tiempo, donde se observa claramente, que la amplitud del estado estable alcanza un valor $X=$ $0.0005694 \mathrm{~m}$ para los datos de la Tabla 3 .

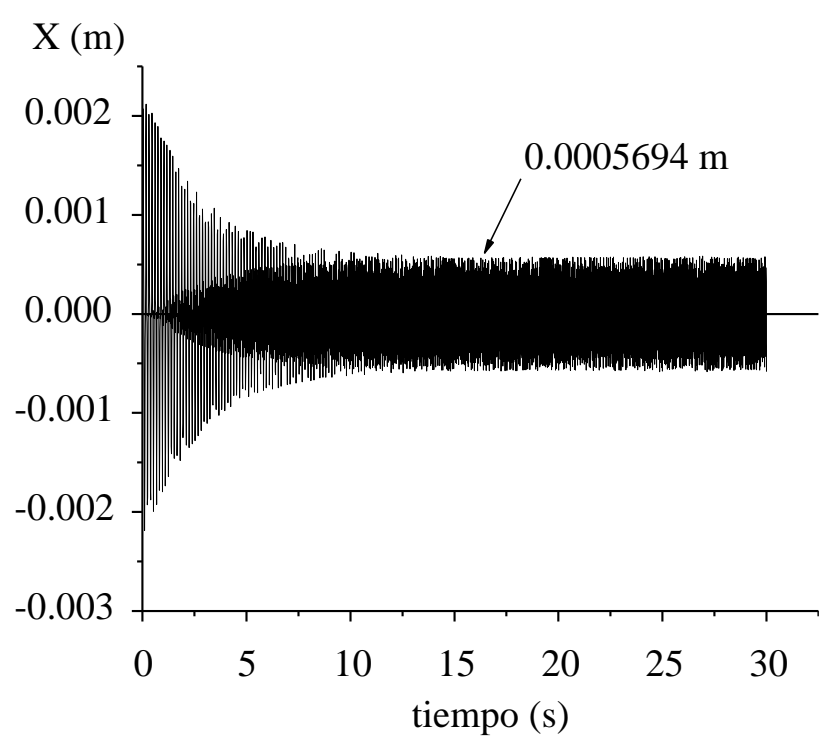

Figura 4 Respuesta en el estado estable para los datos de la Tabla 3

Con los resultados gráficos obtenidos hasta ahora se puede observar lo siguiente, el amortiguamiento entre la simulación de la Figura 3 y la Figura 4, tiene una variación del 20 $\%$, con esta variación del amortiguamiento, se observa que el estado transitorio tiene una duración mayor, por ejemplo, para el valor de $t$ $=10$ seg., la simulación de la Figura 3 se mantiene en el estado estable, mientas que para la Figura 4, en ese instante se mantiene todavía el estado transitorio.

Estos valores numéricos, aunque son muy pequeños están cercanos con lo previsto en la respuesta en la frecuencia, sin embargo, existe una pequeña variación. La respuesta en frecuencia para ambas simulaciones se presenta en la gráfica de la Figura 5.

Es cierto que al disminuir el amortiguamiento, la amplitud tiene a aumentar, sin embargo, una de las características fundamentales del movimiento vibratorio forzado es que para altas frecuencias, los valores de la amplitud tienden a un mismo valor. Como parámetro de referencia para evaluar el comportamiento de las respuestas en el tiempo, se puede emplear la ecuación (7), que permite obtener una representación gráfica en el dominio de la frecuencia. En la Figura 5, se muestra la gráfica de la respuesta en frecuencia para los parámetros de la Tabla 4.

VÁZQUEZ-GONZÁLEZ, Benjamín, JIMÉNEZ-RABIELA, Homero, RAMÍREZ-CRUZ, José Luis y BRAVO-ACOSTA, Adrian Gustavo. Algunos errores numéricos en la respuesta del estado estable de sistemas mecánicos vibratorios. Revista de Ingeniería Mecánica. 2019. 


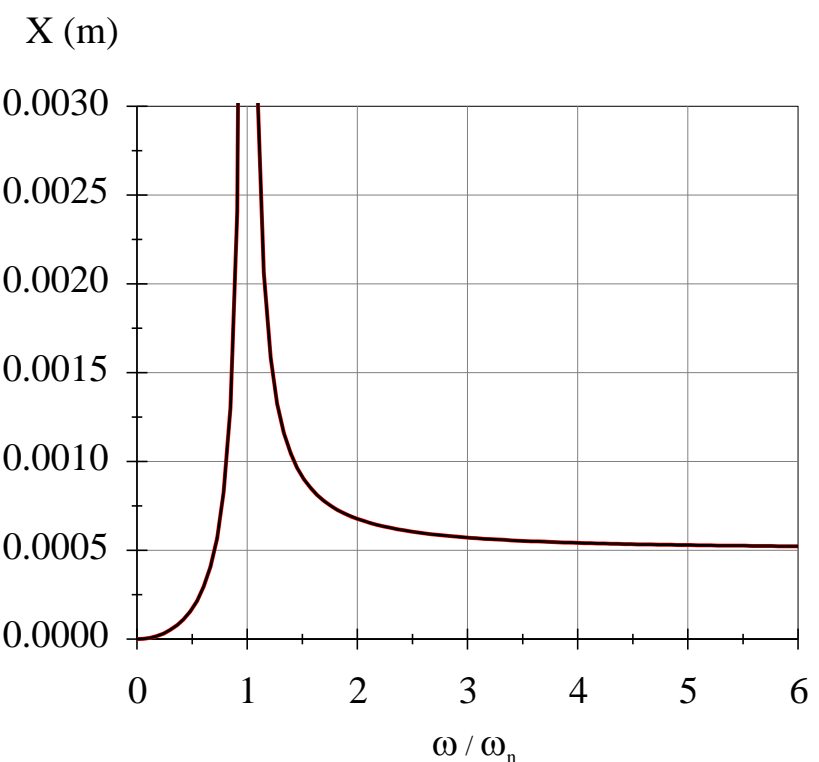

Figura 5 Gráfica de la ecuación (7), para los valores de la Tabla 4

\begin{tabular}{|l|l|}
\hline $\mathrm{m}_{0} \mathrm{r}=0.2303[\mathrm{kgm}]$ & $\mathrm{m}=453.4[\mathrm{~kg}]$ \\
\hline$\zeta=0.01$ & $\zeta=0.008$ \\
\hline
\end{tabular}

Tabla 4 Valores numéricos de los parámetros del sistema masa-resorte amortiguador para la Figura 5

En la gráfica de la Figura 5 se observa que las respuestas son muy semejantes, esto es, $\zeta=0.008$ (color negro) y $\zeta=0.01$ (color rojo), estas dos curvas prácticamente están sobrepuestas. Para apreciar con claridad los valores mencionados con anterioridad, en la siguiente figura se muestra una toma en aumento de la Figura 5.

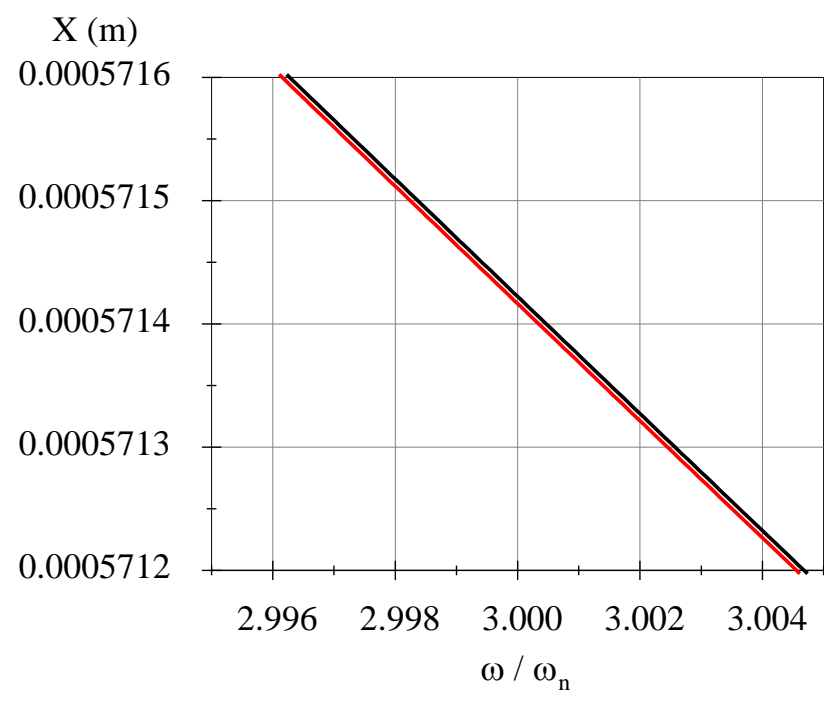

Figura 6 Auemento de la Figura 5 para el rango de 2.996 a 3.004, del cociente de frecuencias $\frac{\omega}{\omega_{n}}$.

Ambos valores de la respuesta en el estado estable, están relativamente alejados de la respuesta esperada conforme la gráfica de la respuesta en frecuencia de la Figura 6, éstos deberían estar por arriba de $\mathrm{X}=0.0005714 \mathrm{~m}$.
En estas condiciones una alternativa para determinar las discrepancias encontradas es utilizar una herramienta de simulación alterna. Para comparar los resultados obtenidos por medio de las simulaciones, a continuación se presenta la gráfica correspondiente a la Tabla 3, obtenida por medio del simulador Matlab ${ }^{\circledR}$. Se ha empleado el método ODE45, basado en el método Runge-Kutta-Fehlberg 4/5.

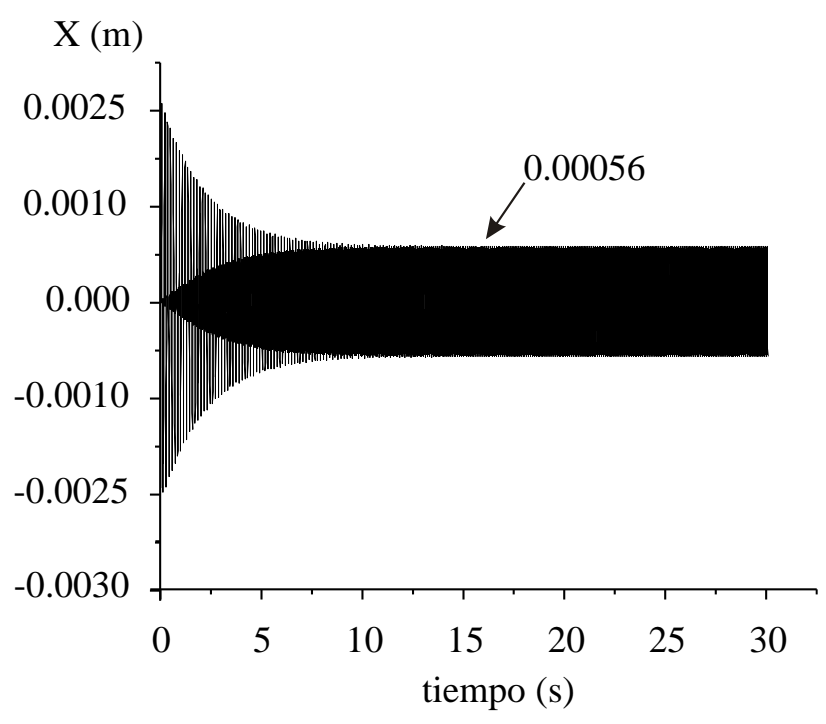

Figura 7 Respuesta en el estado estable para los datos de la Tabla 3, utilizando ODE45 de Matlab

La Figura 3 y la Figura 7 reportan prácticamente el mismo resultado. Las gráficas mostradas en esta sección tienen como objetivo ilustrar la consistencia aparente entre un resultado obtenido por medio de una simulación numérica y la respuesta en el estado estable, proporcionada por la solución analítica. Un investigador podrá considerar que el método empleado en la simulación numérica es confiable, porque las respuestas determinadas por ambos esquemas son semejantes.

El objetivo de este trabajo es mostrar la discrepancia que puede existir, cuando se elige un método numérico que proporciona valores semejantes, en un rango pequeño de respuestas, sin que el investigador note los grandes errores que se pueden presentar.

\section{Determinación de los errores en las simulaciones numéricas}

En esta sección se estudia un caso representativo de errores que se pueden presentar, en el desarrollo de simulaciones numéricas. 
Hasta ahora existe congruencia entre las simulaciones realizadas por el método numérico Runge-Kutta-Fehlberg 4/5 en ambos simuladores, que presentan respuestas en el tiempo y la gráfica de la respuesta en frecuencia, que muestra la amplitud en el estado estable.

Con los resultados obtenidos se puede considerar que los resultados posteriores estarán siendo representados consistentemente para cualquier simulación futura.

Al realizar simulaciones posteriores, empleando los mismos simuladores y los mismos métodos numéricos se ha detectado que, lo anterior es válido sólo para algunas condiciones.

Estas condiciones no son evidentes, cambios posteriores en algunos de los parámetros de las simulaciones anteriores llevan a respuesta que pueden ser erróneas, y llevar a conclusiones incorrectas.

Considérense ahora los parámetros de la siguiente tabla.

\begin{tabular}{|l|l|}
\hline$\omega=3 \omega_{\mathrm{n}}[\mathrm{rad} / \mathrm{s}]$ & $\mathrm{m}_{0} \mathrm{r}=0.2303[\mathrm{kgm}]$ \\
\hline$\omega_{\mathrm{n}}=44[\mathrm{rad} / \mathrm{s}]$ & $\mathrm{m}=453.4[\mathrm{~kg}]$ \\
\hline$\zeta=0$ & \\
\hline
\end{tabular}

Tabla 5 Valores numéricos de los parámetros para la Figura 8

Con los parámetros de la Tabla 5 se realiza una simulación conforme al método RKF4/5. El resultado se muestra en la Figura 8.

A diferencia de las simulaciones numéricas en función del tiempo, ahora el rango del mismo se extiende hasta $t=300 \mathrm{seg}$. No se considera la presencia de amortiguamiento, pero se tiene una relación de frecuencias de $\frac{\omega}{\omega_{n}}=3$.

El valor esperado de la amplitud, conforme la respuesta en la frecuencia (ver Figura 6) debe ser aproximadamente de $X=$ $0.000571 \mathrm{~m}$, sin embargo, el resultado es completamente distinto. Cómo se mencionó con anterioridad, para altas frecuencias todos los valores de la amplitud convergen a un mismo valor, incluyendo el caso no amortiguado, se observa una respuesta completamente distinta y errónea de la considerada previamente.
Las simulaciones anteriores estaban restringidas para un rango de tiempo de hasta $t=$ 30 seg. Al ampliar el rango del tiempo de la simulación, utilizando el mismo método numérico (RGK4/5), se aprecia que las primeras simulaciones sólo ofrecían un resultado parcial.

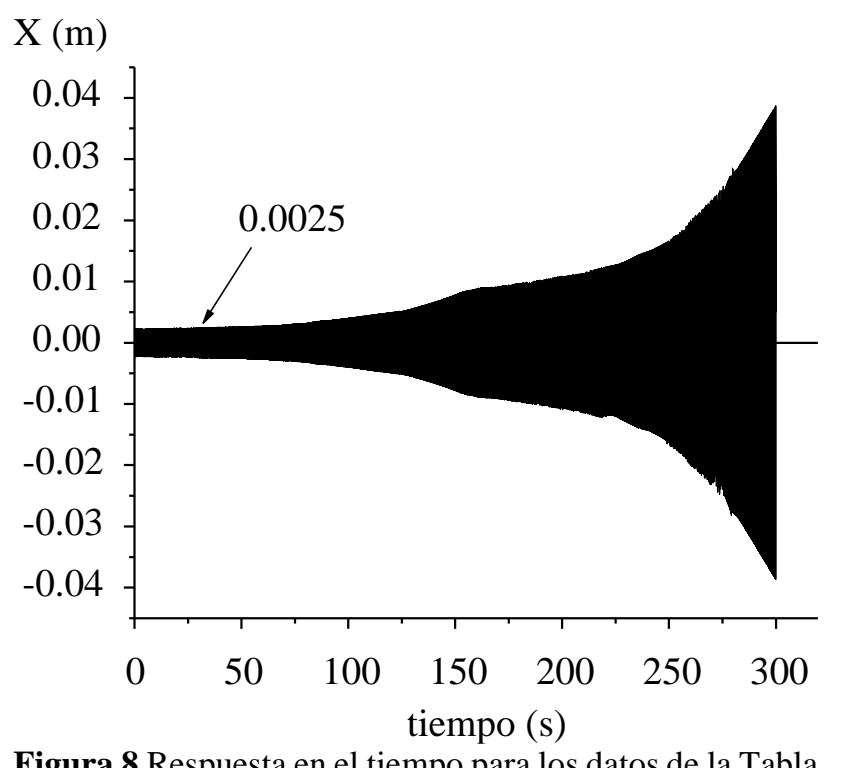

Figura 8 Respuesta en el tiempo para los datos de la Tabla 5

Para descartar un error atribuible al simulador, se decidió, realizar una simulación idéntica a la anterior, pero con el simulador ODE45 de Matlab ${ }^{\circledR}$ y el resultado es semejante, se reportan valores no consistentes con la respuesta en frecuencia.

Con el fin de determinar la falta de consistencia atribuible al comportamiento del sistema bajo las condiciones impuestas, se realiza el siguiente experimento numérico. Considérese ahora los parámetros de la siguiente tabla.

\begin{tabular}{|l|l|}
\hline$\omega=6 \omega_{\mathrm{n}}[\mathrm{rad} / \mathrm{s}]$ & $\mathrm{m}_{0} \mathrm{r}=0.2303[\mathrm{kgm}]$ \\
\hline$\omega_{\mathrm{n}}=44[\mathrm{rad} / \mathrm{s}]$ & $\mathrm{m}=453.4[\mathrm{~kg}]$ \\
\hline$\zeta=0$ & \\
\hline
\end{tabular}

Tabla 6 Valores numéricos de los parámetros para la Figura 9

El resultado de la simulación numérica correspondiente a los datos de la Tabla 6, se presenta la Figura 9. Ahora se presenta nuevamente el comportamiento típico de la respuesta de la vibración forzada, se aprecia claramente el estado estable, sin embargo, la única diferencia entre las gráficas de las Figuras 8 y 9 es el rango de frecuencia, en la Figura 9, la respuesta corresponde a una relación en frecuencia $\frac{\omega}{\omega_{n}}=6$. 
El único referente concreto es la gráfica de la Figura 6, que contiene la respuesta completa de la amplitud. Por lo que la conclusión hasta ahora es que el método numérico puede dar resultados incorrectos. Es importante mencionar en este momento, que sin el conocimiento previo de la respuesta de la amplitud en el contexto de la frecuencia, el resultado obtenido a partir de la simulación numérica se puede considerar como correcto, y llegar a la conclusión de que el método numérico, puede producir errores no es inmediato, porque los método numéricos son herramientas que se consideran confiables.

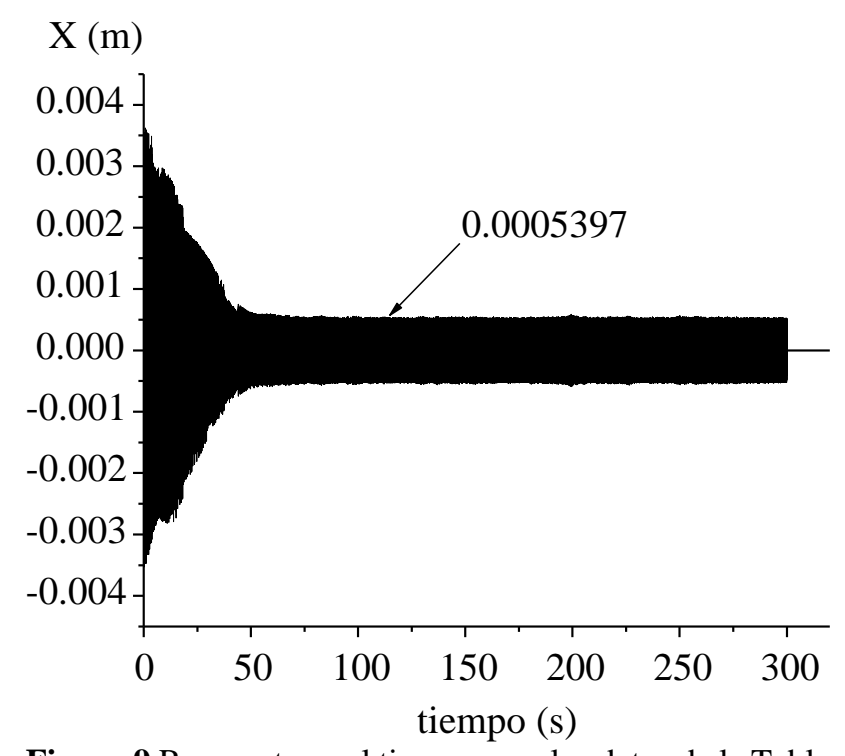

Figura 9 Respuesta en el tiempo para los datos de la Tabla 3

Con el fin de validar la conclusión anterior, en la Figura (10) se presenta una simulación realizada con los datos de la Tabla 5, pero utilizando el método numérico RungeKutta-Fehlberg 2/3.

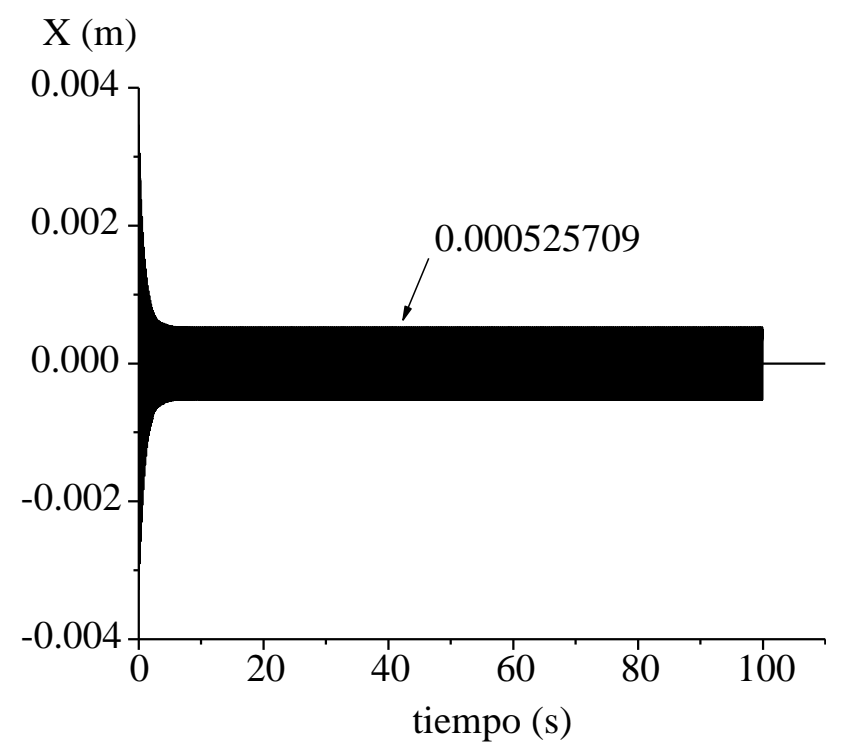

Figura 10 Respuesta en el tiempo para los datos de la Tabla 5, utilizando el método Runge-Kutta-Fehlberg 2/3
Se observa que la respuesta de la gráfica se presenta con mayor definición. Esta respuesta es consistente con lo preestablecido por la respuesta en frecuencia, el amortiguamiento es nulo y el estado transitorio es muy corto, debido a que al amortiguamiento es nulo.

\section{Conclusiones}

En este trabajo se ha revisado una de las situaciones más comunes en el análisis de la vibración mecánica, se trata del movimiento armónico forzado amortiguado. Existen dos herramientas básicas para éste análisis, una es la respuesta en la frecuencia que corresponde con resolver la ecuación diferencial asociada y expresar la solución en términos de la frecuencia de excitación, esto proporciona la respuesta para el caso general, sin embargo, reconstruir la respuesta exacta a partir de la respuesta en frecuencia puede llevar a obtener respuestas aproximadas en el tiempo.

La otra herramienta es la que proporcionan los métodos numéricos, en este caso, el método numérico empelado puede llevar a resultados incorrectos cuando el método elegido no es el adecuado.

Aquí se ha presentado un caso en el que se muestra que no se cumple la condición de estado estable con un método numérico particular, sin embargo, no es fácil determinar en qué condiciones el método ha proporcionado la solución correcta, sí se elige un rango de tiempo relativamente corto, entonces no se apreciará el error y se considerará que la solución es correcta para cualquier lapso de tiempo. Cuando se combinan las dos herramientas, entonces es posible garantizar el desempeño del sistema para cualquier rango de tiempo, conforme lo predice la respuesta en frecuencia y con un método confiable. La simulación numérica en el análisis de las vibraciones mecánicas requiere de muchos recursos informáticos, porque se acumula una gran cantidad de datos, esto puede producir fallas en el método numérico que se utilice.

\section{Agradecimiento}

Los autores agradecemos el apoyo institucional, de la Universidad Autónoma Metropolitana y de la Unidad Azcapotzalco, en particular de la División de Ciencias Básicas e Ingeniería y del Departamento de Energía. 


\section{Referencias}

Åström, Karl Johan. (1982). A SIMNON Tutorial. Department of Automatic Control, Lund Institute of Technology (LTH), (Research Reports TFRT-3168).

Chunling Du and Chee Khiang Pang, MultiStage Actuation Systems and Control. 2019 by Taylor \& Francis Group.

Emerson, High Frequency Vibration Analysis, AMS 2140 Machinery Health Analyzer. White Paper December 2017. (https://www.emerson.com/documents/automati on/white-paper-high-frequency-vibrationanalysis-ams-en-39104.pdf)

Ginsberg, Jerry H, Mechanical and Structural Vibrations. Theory and Applications. First Edition, 2001. John Wiley \& Sons, Inc.

León, R. A. G., Solan, E. F., \& Pedroza, J. (2019). Diseño de un banco de pruebas para el análisis de vibraciones mecánicas. Revista Colombiana de Tecnologías Avanzadas (RCTA), 1(33).

MATLAB $^{\circledR}$ High-Performance Numeric Computation and Visualization Software. Refernce Guide. The MathWorks Inc

Meirovitch, Leonard. Fundamentals of Vibrations. McGraw-Hill, 2001. 